\title{
Detection of Truncated Circular DNA Species in Escherichia coli with a PCV2-Containing Plasmid with a Single PCV2 Origin of Replication
}

\author{
J. Van Doorsselaere ${ }^{a}$ D.J. Lefebvre ${ }^{b}$ H.J. Nauwynck ${ }^{b}$ P.L. Delputte ${ }^{b}$

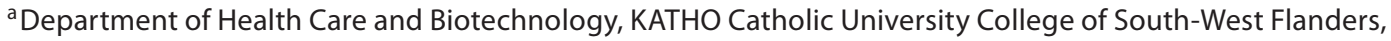 \\ Roeselare, and ${ }^{b}$ Department of Virology, Parasitology and Immunology, Faculty of Veterinary Medicine, \\ Ghent University, Merelbeke, Belgium
}

\section{Key Words}

Porcine circovirus type $2 \cdot$ Escherichia coli Infectious clone · Truncation of plasmid DNA

\begin{abstract}
Objective: Porcine circovirus type 2 (PCV2) is a small circular single-stranded DNA virus that causes postweaning multisystemic wasting syndrome in pigs. Cloning of the PCV2 genome in a plasmid allows the construction of infectious clones. Our objective was to clone single PCV2 genomes from an isolate containing a mixture of strains, in a plasmid in order to obtain pure PCV2 strains. Methods: PCR amplification of PCV2 genomes and cloning followed by restriction enzyme analysis and sequencing. Transfection and PCV2 titration on PK-15 cells. Results: Single-copy PCV2 genomes from three Belgian PCV2 strains were cloned. Unexpectedly, agarose gel analysis revealed that additional circular DNA species were generated in Escherichia coli. Restriction enzyme analysis and sequencing showed that the circular DNA species were truncated and derived from the plasmid containing the PCV2 genome. Mutagenesis of the PCV2 replicase gene abolished the formation of these DNA species. The infectious clones were transfected in PK-15 cells and pure PCV2 viral strains were obtained. Conclusion: Infectious clones were obtained that can be used for antigenic
\end{abstract}

mapping and mutagenesis. In addition, our findings suggest that the replicase protein was expressed in E. coli and involved in the generation of the truncated DNA species.

Copyright $\odot 2009$ S. Karger AG, Basel

\section{Introduction}

Porcine circovirus type 2 (PCV2) is a member of the genus Circovirus of the family Circoviridae [1]. PCV2 is a ubiquitous pathogen in pigs, and under certain conditions PCV2 infection may cause postweaning multisystemic wasting syndrome, a multifactorial disease that causes severe growth retardation, weight loss and death in weaned pigs [2-4]. PCV2 is a nonenveloped virus with a circular, single-stranded DNA (ssDNA) of approximately $1.77 \mathrm{~kb}$. The ambisense genome has 2 major open reading frames (ORFs). ORF1 encodes 2 replicase proteins (Rep and Rep') [5]. ORF2 codes for the structural capsid protein [6]. The origin of replication (Ori), which is used in rolling circle replication (RCR) of the genome during virus replication, is situated between these ORFs [7].

The small size of the PCV genome easily allows construction of infectious clones by PCR amplification and consequent cloning of the full-length genome in a plas-

Jan Van Doorsselaere

Department of Health Care and Biotechnology

KATHO Catholic University College of South-West Flanders

Wilgenstraat 32, BE-8800 Roeselare (Belgium)

Tel. +32 512323 30, Fax +32 512282 58, E-Mail jan.vandoorsselaere@ katho.be 
mid. Two strategies have been described for the construction of infectious clones. Lekcharoensuk et al. [8] cloned one PCV genome copy in a plasmid. To generate infectious virus, the full-length PCV genome has to be excised using restriction enzymes followed by in vitro transfection of PK-15 cells. Another approach is the insertion of more than one genome copy in tandem in a plasmid [912]. The advantage of this strategy is that these plasmids can be directly transfected in PK-15 cells to generate infectious virus. This is due to the presence of two PCV Oris in the plasmid, which allows the PCV RCR to occur in transfected cells starting from the circular plasmid. Surprisingly, RCR was also shown to take place in Escherichia coli using the in E. coli expressed PCV Rep gene and the two PCV Oris. As in PK-15 cells, infectious DNA is produced in $E$. coli $[12,13]$.

Previously, Lefebvre et al. [14] reported the full-length genome sequences of three Belgian PCV2 strains (II11A, II9F and 4D4) of which strain 4D4 was a result of recombination between II11A and II9F. To be able to study the in vitro characteristics of clearly defined clones of these three Belgian PCV2 strains, and not potential mixtures of these strains, infectious clones were generated by cloning a single PCV2 genome in a plasmid. Unexpectedly, propagation of the plasmid containing the single-copy PCV2 genome in E. coli resulted in the formation of additional circular DNA species. Restriction enzyme analysis and sequencing showed that these fragments were generated from the PCV2-containing plasmid and the involvement of Rep in the generation of these additional DNA species was examined.

\section{Materials and Methods}

Construction of Single-Copy PCV2 Infectious Clones

The full-length genomes of PCV2 strains II11A (EU909686), 4D4 (EU909687) and II9F (EU909688) [14] were amplified by PCR using primers INFCL-PCV2-FW (5'-GGAAGCTTCAGTAATTTATTTCATATGGAA-3') and INFCL-PCV2-REV (5'GGAAGCTTTTTTATCACTTCGTAATGGTT-3'), hereby introducing a HindIII site (underlined in the primer sequence) after the stop codon of the Rep gene. The PCV2 genomes were each cloned in pCR-BluntII-TOPO (Invitrogen, Merelbeke, Belgium) after position 336 of that vector. The sequence of pCR-BluntIITOPO is available at www.invitrogen.com. The recombinant plasmids (fig. 1) were transformed in E. coli strains DH5alfa or TOP10. Plasmid DNA was extracted using a plasmid DNA kit (Qiagen Benelux, Venlo, The Netherlands). Agarose gel electrophoresis and restriction enzyme analysis were performed according to standard procedures.
Sequence Analysis

The sequences of the PCV2 genomes in the plasmid were determined (using M13FW, M13REV and PCV2-specific primers $[14,15])$ and were found to be identical with the genomic sequences of II11A, 4D4 and II9F, except for 4D4 clone 5, which had 3nucleotide (nt) differences with EU909687. This resulted in an amino acid change at position $80(\mathrm{R} \rightarrow \mathrm{C})$ in the Rep protein. This aa position is located outside the four functional domains of the Rep [16]. All clones had the HindIII site after the stop codon of the Rep gene.

Additional DNA species were gel purified using a QiaQuick gel extraction kit (Qiagen Benelux) and sequenced directly as described above.

\section{Mutagenesis of the Rep Gene}

Mutagenesis of $\mathrm{Y}^{93}$ in motif III of the Rep gene (an amino acid that is essential for nicking activity [16]), was performed using a Quickchange site-directed mutagenesis kit (Stratagene, La Jolla, Calif., USA) with primers motIIIFW (5'-TCAGCAGAATAAAGAATTTTGCAGTAAAGAAGGCA-3') and motIIIREV (5'-TGCCTTCTTTACTGCAAAATTCTTTATTCTGCTGA-3').Underlined is the $\mathrm{F}^{93}$ codon. Plasmid DNA was isolated from several clones and sequenced.

\section{Results}

Cloning of Single-Copy Infectious Genomes and Detection of Additional DNA Species in E. coli Harboring a pCR-BluntII-TOPO Plasmid with One

\section{PCV2 Genome}

Lefebvre et al. [14] reported the full-length sequences of three PCV2 strains (II11A, II9F and 4D4). Using these strains, single-copy infectious PCV2 genomes were cloned into pCR-BluntII-TOPO. The sequences of the PCV2 genomes and the orientation of the PCV2 genomes in the plasmid were determined. In figure 1, a plasmid map of an infectious clone is shown.

Plasmid DNA from various clones was analyzed using agarose gel electrophoresis. An overview of analyzed clones is given in table 1 . In addition to the plasmid containing the PCV2 genome (seen as two bands on a gel representing 4-kbp supercoiled and 8 -kbp relaxed plasmid, respectively), other DNA species (or bands) were detected in several but not all clones (fig. 2). The size of these additional DNA species was between 1.5 and $1.7 \mathrm{kbp}$. Some clones contained more than one additional DNA species (e.g. II9F clone 18). Sequencing of the clones with additional DNA species revealed that all these had the same orientation of the PCV2 genome in the vector, as shown in figure 1 . In II9F clone 14, additional DNA species were not detected despite the fact that the PCV2 genome had the same orientation as 
Table 1. Overview of analyzed original clones, derived truncated plasmids and clones with mutagenized Rep

\begin{tabular}{|c|c|c|c|c|c|c|}
\hline \multirow[t]{2}{*}{ Clone name } & \multicolumn{3}{|c|}{ Nondigested } & \multicolumn{3}{|c|}{ HindIII digested } \\
\hline & figure & lane & fragment sizes, $\mathrm{kbp}$ & figure & lane & fragment sizes, kbp \\
\hline II11A clone 1 & $2 \mathrm{a}$ & 2 & 8 and 4 & $2 \mathrm{~b}$ & 2 & $3,1.8$ and $0.065^{\mathrm{a}}$ \\
\hline $4 \mathrm{D} 4$ clone 10 & $2 \mathrm{a}$ & 6 & 8,4 and $1.5-1.7^{b}$ & $2 \mathrm{~b}$ & 3 & $3,2.7,1.8,1$ and 0.065 \\
\hline II9F clone 13 & $2 \mathrm{a}$ & 8 & 8,4 and $1.5-1.7$ & $2 \mathrm{~b}$ & 4 & $3,2.7,1.8$ and 0.065 \\
\hline II9F clone 14 & $2 \mathrm{a}$ & 9 & 8,4 and $1.5-1.7$ & $2 \mathrm{~b}$ & 5 & $3,1.8$ and 0.065 \\
\hline II9F clone 18 & $2 \mathrm{a}$ & 10 & 8,4 and $1.5-1.7$ & ND & ND & ND \\
\hline II9F clone 23 & $2 \mathrm{a}$ & 12 & 8,4 and $1.5-1.7$ & ND & ND & ND \\
\hline Tr4D4 clone 10 (several clones) & $5 a$ & $3-6$ & 1.7 & $5 b$ & $3-6$ & 2.7 and 0.065 \\
\hline TrII9F clone 18 (several clones) & $5 a$ & $9-13$ & 1.5 or 1.7 & $5 b$ & $9-13$ & (2.5 or 2.7$)$ and 0.065 \\
\hline Rep mutagenized II9F clone 13 & $6 a$ & $3-7$ & 8 and 4 & $6 c$ & $3-7$ & $3,1.8$ and 0.065 \\
\hline Rep mutagenized II9F clone 23 & $6 a$ & $10,11,13,14$ & 8 and 4 & $6 c$ & $10,11,13,14$ & $3,1.8$ and 0.065 \\
\hline \multirow[t]{2}{*}{ Rep nonmutagenized II9F clone 23} & $6 a$ & 12 & 8 and 4 & $6 c$ & 12 & $3,2.7,1.8$ and 0.065 \\
\hline & $6 \mathrm{~b}$ & 3 & 8,4 and $1.5-1.7$ & & & \\
\hline
\end{tabular}

ND $=$ Not done.

${ }^{a}$ In figure $2 \mathrm{~b}$ the $0.065-\mathrm{kbp}$ fragments are not seen on the gel due to the fact that only fragments $>0.3 \mathrm{kbp}$ are shown.

b The additional DNA species (1.5-1.7 kbp) from 4D4 clone 10 and II9F clone 18 (indicated in bold) were gel purified and transformed in E. coli. This resulted in a number of truncated clones (Tr4D4 clone 10 and TrII9F clone 18).

shown in figure 1 . No additional bands were seen in the clones with the PCV2 genome in the opposite orientation.

Subsequently, the clones were digested with HindIII in order to release the PCV2 genome. A HindIII digest should result in three fragments with approximate sizes of $3 \mathrm{kbp}$ (pCR-BluntII-TOPO vector), $1.8 \mathrm{kbp}$ (PCV2 insert) and $0.065 \mathrm{kbp}$ (fig. 1; table 1). These three fragments were seen in all clones, but an additional fragment of 2.7 $\mathrm{kbp}$ was present in clones with additional DNA species, suggesting that the additional $1.5-$ to $1.7-\mathrm{kbp}$ DNA species were supercoiled plasmids of size $2.7 \mathrm{kbp}$ containing HindIII restriction sites (fig. 2). In 4D4 clone 10, an additional 1-kbp HindIII fragment was present (but this was not further investigated).

The HindIII-digested plasmids were transfected into PCV-negative PK-15 cells with Lipofectamine (Invitrogen), according to the manufacturer's instructions. Subsequently, PCV2 was passed several times on PK-15 cells. Indirect immunofluorescence staining of PCV2-infected PK-15 cells and titration of supernatant fluids from PCV2-infected PK-15 cells on PCV-negative PK-15 cells demonstrated that PCV2 virus was produced and that infectious clones had been produced (data not shown). PCV2 sequences were obtained after five passages on PK15 cells and sequences corresponded with the sequences of II11A, II9F and 4D4.

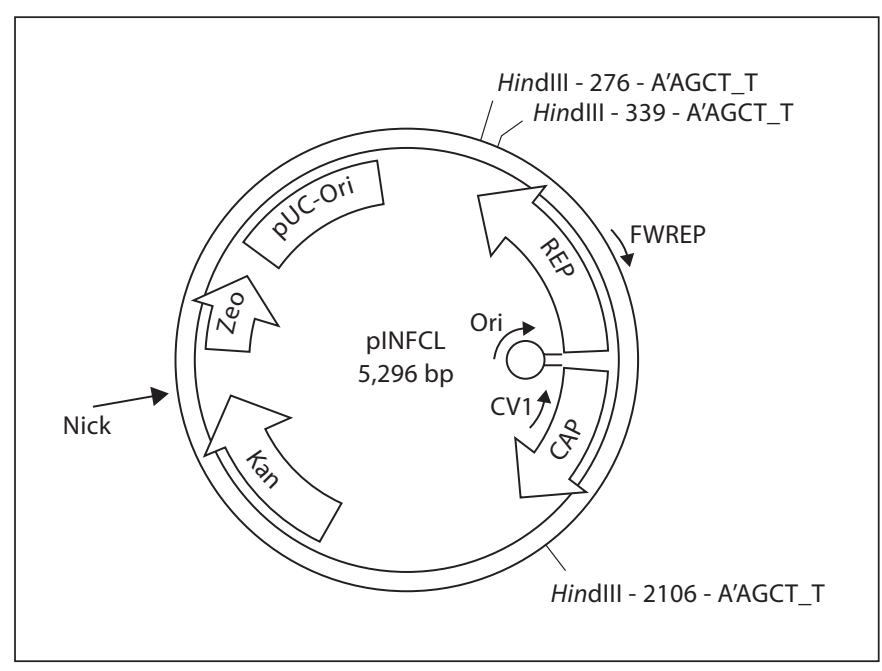

Fig. 1. Representation of the PCV2 genome in pCR-BluntII-TOPO. The direction of the PCV2 Ori is indicated. The position and direction of the PCV2-specific sequencing primers CV1 [15] and FWREP [14] are shown.

\section{Identities of the Additional DNA Species}

Cheung [12] demonstrated that the Rep protein is produced and initiates RCR in E. coli. The presence of the additional $2.7-\mathrm{kbp}$ band observed in this study may be explained if the additional DNA species would result 


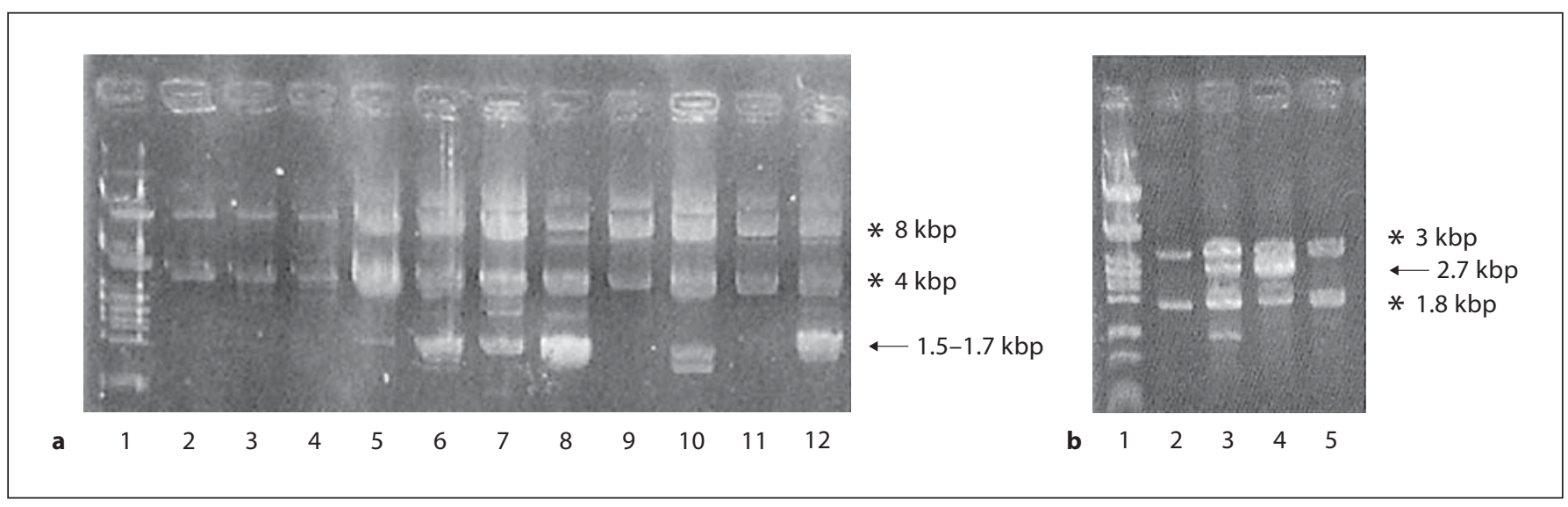

Fig. 2. Agarose gel electrophoresis of plasmid DNA from nondigested DNA (a) and HindIII-digested DNA (b) from PCV2 infectious clones. a Plasmid DNA containing one PCV2 genome can be seen in all lanes as two bands (upper asterisk: relaxed plasmid; lower asterisk: supercoiled plasmid). Additional DNA bands (arrow) between 1.5 and $1.7 \mathrm{kbp}$ can be seen in lanes 6 (4D4 clone 10), 7 (4D4 clone 11), 8 (II9F clone 13), 10 (II9F clone 18) and 12

from RCR starting at the PCV2 Ori, followed by a nick in the pCR-BluntII-TOPO plasmid around the start of the Zeocin resistance (ZeoR) gene and subsequent ligation, resulting in truncated plasmids without the kanamycin resistance (KanR) gene and the PCV2 capsid gene.

In order to test this hypothesis, the additional DNA species present in several clones were gel purified and sequenced using primers CV1 and FWREP flanking the Ori $[15,14]$. For convenience, the truncated plasmids were named $\operatorname{Tr} 4 \mathrm{D} 4$ clone $10, \operatorname{Tr} 4 \mathrm{D} 4$ clone 11 , etc. Sequencing with primer CV1, which hybridizes with the PCV2 capsid gene, did not result in sequence data, suggesting that the PCV2 capsid gene was not present in the truncated plasmids. Sequencing with primer FWREP, which hybridizes with the PCV2 Rep gene, was successful in all cases, and the chromatogram of $\operatorname{Tr} 4 \mathrm{D} 4$ clone 11, TrII9F clone 13 and TrII9F clone 23 yielded nonambiguous sequence data. The chromatogram of $\mathrm{Tr} 4 \mathrm{D} 4$ clone 10 and TrII9F clone 18 contained a mixed peak pattern, showing that more than one DNA species was present. This was already evident for TrII9F clone 18, as noticed by different bands observed in agarose gel (fig. 2).

An overview of the sequences from the truncated clones is given in figure $3 \mathrm{~b}$. Only the region where the plasmid has been nicked and joined to the PCV2 Ori is shown. A graphical representation of a truncated plasmid is shown in figure 4. The clones Tr4D4 clone 11 and $\operatorname{Tr} 4 \mathrm{D} 4$ clone 13 were identical and had a nick after posi-
(II9F clone 23). More than one additional DNA species can be seen in lane 10. Lane 1: $\lambda$ PstI ladder. b HindIII digest of a selection of clones without additional DNA species (lanes 2 and 5) and with additional DNA species (lanes 3 and 4). An extra band of $2.7 \mathrm{kbp}$ (arrow) is present in lanes 3 and 4 . In all cases, a $0.065-\mathrm{kbp}$ fragment was present. This band is not seen on the gel since only fragments $>0.3 \mathrm{kbp}$ are shown. Lane 1: $\lambda$ Pst I ladder.

tion 2051 in pCR-BluntII-TOPO (with the position of the ZeoR gene from 2238 to 2612). In the region around the nick in the plasmid, several repeats were present: an inverted repeat of $6 \mathrm{nt}^{2080}$ [GATGCG(14 bp)CGCATC] ${ }^{2055}$ and an overlapping direct repeat of $10 \mathrm{nt}^{2092}$ [AAATACCGCA(16 bp)AAATACCGCA $]^{2057}$ before the nick and a direct repeat of $10 \mathrm{nt}{ }^{2015}$ [AGAAGGCGAT(0 bp)AGAAGGCGAT] ${ }^{1996}$ after the nick (not shown in fig. 3). In TrII9F clone 23, the nick occurred between positions 1713 and 1719 in the plasmid. An inverted repeat of $6 \mathrm{nt}$ ${ }^{1769}$ [GCTCGC(28 bp)GCGTGC] ${ }^{1730}$ was present before the nick in the plasmid.

As can be seen in figure 4, the truncated plasmids lacked the KanR gene but still had the ZeoR gene. Therefore, Tr4D 4 clone 10 and TrII9F clone 18, which contained more than one plasmid (as noticed by agarose gel electrophoresis and by the mixed peak pattern in the chromatogram), were transformed in E. coli. Transformed colonies were obtained on medium with Zeocin but no colonies grew on medium with kanamycin, suggesting that these clones did not contain the KanR gene and that truncation also occurred upstream of the ZeoR gene.

Plasmid DNA from nine ZeoR clones, obtained by transforming Tr4D4 clone 10 or TrII9F clone 18, was analyzed by agarose gel electrophoresis (fig. 5). For Tr4D4 clone 10, all DNA species had a size of approximately 1.5 $\mathrm{kbp}$. For TrII9F clone 18, DNA species were 1.5 or $1.7 \mathrm{kbp}$, 
Fig. 3. a Representation of the PCV2 Ori. The Oc8 sequence is present in the stemloop $(\mathrm{A} \times \mathrm{TA} \times \mathrm{T} \downarrow \mathrm{AC})$. The hexamer repeats $\mathrm{H} 1-\mathrm{H} 4$ are underlined. b Partial sequences of truncated clones $\mathrm{Tr} 4 \mathrm{D} 4$ clone 11, TrII9F clone $18 \mathrm{a}$, TrII9F clone $18 \mathrm{~b}$ and TrII9F clone 23. The sequences on the right correspond with PCV2 Ori sequences; the sequences on the left correspond with sequences from pCR-BluntII-TOPO. The arrow indicates the plasmid-PCV2 transition. Direct repeats are underlined. Inverted repeats are in italics and in bold.

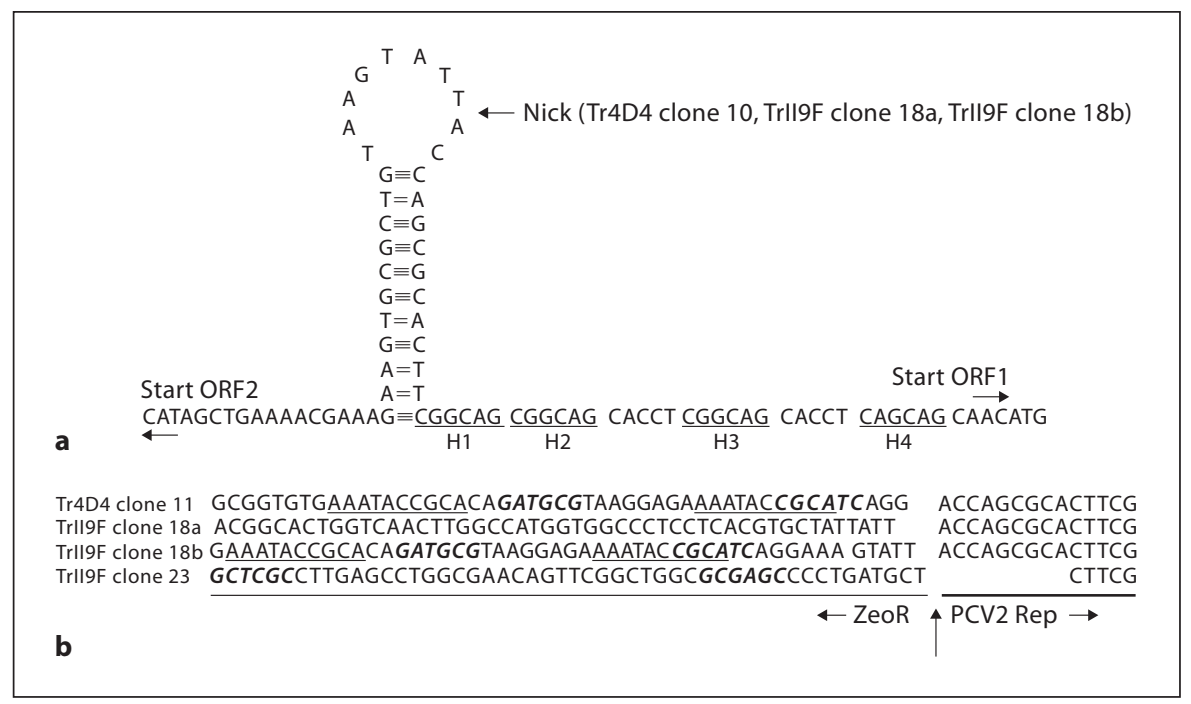

respectively. Truncated clones were sequenced using primer FWREP. Sequences of the five Tr4D4 clone 10 plasmids were identical with $\operatorname{Tr} 4 \mathrm{D} 4$ clone 11. In TrII9F clone 18b, the nick occurred after position 2048 of the plasmid, and $5 \mathrm{nt}$ (GTATT) were present between the nick in the plasmid and the PCV2 Ori (fig. 3b). In TrII9F clone 18 a, the nick occurred after position 2211. No apparent repeats were observed in this region.

\section{Involvement of Rep in the Generation of Additional DNA Species}

To investigate the potential involvement of Rep protein nicking activity in the formation of the additional DNA species, the residue $\mathrm{Y}^{93}$ in the Rep protein motif III was mutagenized to F. $\mathrm{Y}^{93}$ was previously shown to be essential for nicking activity [16]. Nine mutagenized clones, containing the Y-F mutation, did not show any additional bands on agarose gel (fig. 6a). One clone did not contain the Y-F mutation and showed faint additional DNA species (fig. 6b), suggesting that Rep protein nicking activity was essential for the formation of additional DNA species.

\section{Discussion}

In the present study, the construction of infectious PCV2 clones, by introducing a single PCV2 genome copy in a bacterial plasmid, is described. Upon transformation of the plasmids in bacteria, other circular DNA species were generated besides the original plasmid. These DNA

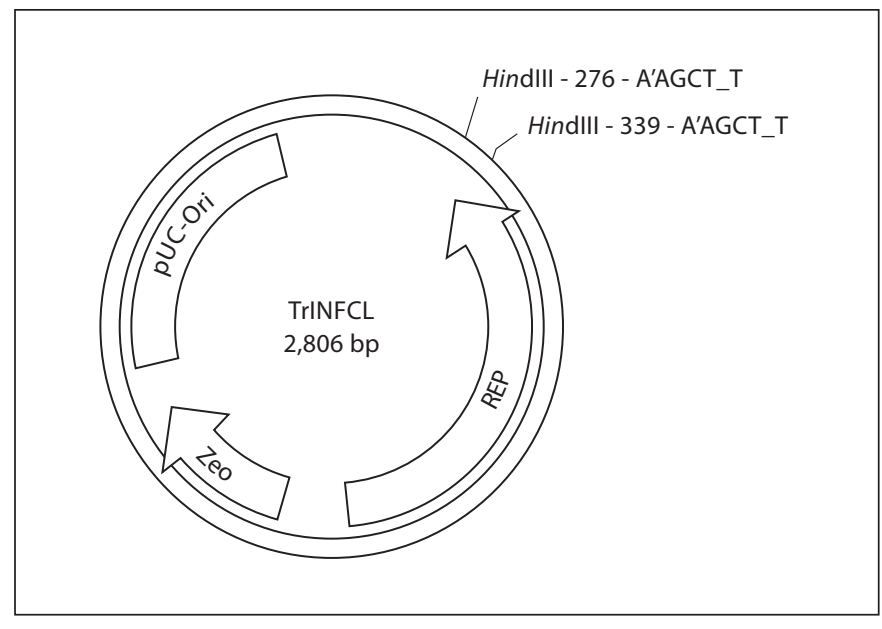

Fig. 4. Representation of a truncated plasmid (e.g. Tr4D4 clone 11). The KanR gene and the PCV2 capsid gene were not present. The size of this plasmid was $2,806 \mathrm{bp}$. Linearization of this plasmid with HindIII resulted in two fragments of approximately 65 and $2,740 \mathrm{bp}$.

species were shown to be truncated forms of the original PCV2-containing plasmids, consisting of a part of the PCV2 Ori with the PCV2 Rep gene and a part of the pCRBluntII-TOPO vector including the ZeoR gene (fig. 4). The construction of PCV infectious clones containing one or more PCV genomes has been reported in the past [8-12], but to our knowledge, additional DNA species were not reported. The data from the present study suggest that the observed truncated plasmids might have 
Fig. 5. a Agarose gel electrophoresis of plasmid DNA from Tr4D4 clone 10 and TrII9F clone 18. Lanes 1 and 7: $\lambda$ PstI; lanes 2 and 8 contain the original clones 4D4 clone 10 and II9F clone 18, respectively. Plasmid DNA containing one PCV2 genome can be seen as two bands (upper asterisk: relaxed plasmid; lower asterisk: supercoiled plasmid), and additional DNA species of 1.5-1.7 kbp are indicated with an arrow. Lanes 3-6 contain a band of approximately $1.5 \mathrm{kbp}$, corresponding with truncated plasmids originating from 4D4 clone 10. Lanes 9-13 contain truncated plasmids originating from II9F clone 18 . Two different band sizes of 1.5-1.7 kbp can be observed. b HindIII digest of the clones from a. The truncated clones (lanes 3-6) derived from the original clone 4D4 clone 10 (lane 2) show one $2.7-\mathrm{kbp}$ fragment (arrow) and a $0.065-\mathrm{kbp}$ fragment. The truncated clones (lanes 9-13) derived from the original clone II9F clone 18 (lane 8) show one 2.5- or 2.7-kbp fragment (arrows) and a 0.065-kbp fragment. Lanes 1 and 7: $\lambda$ PstI.

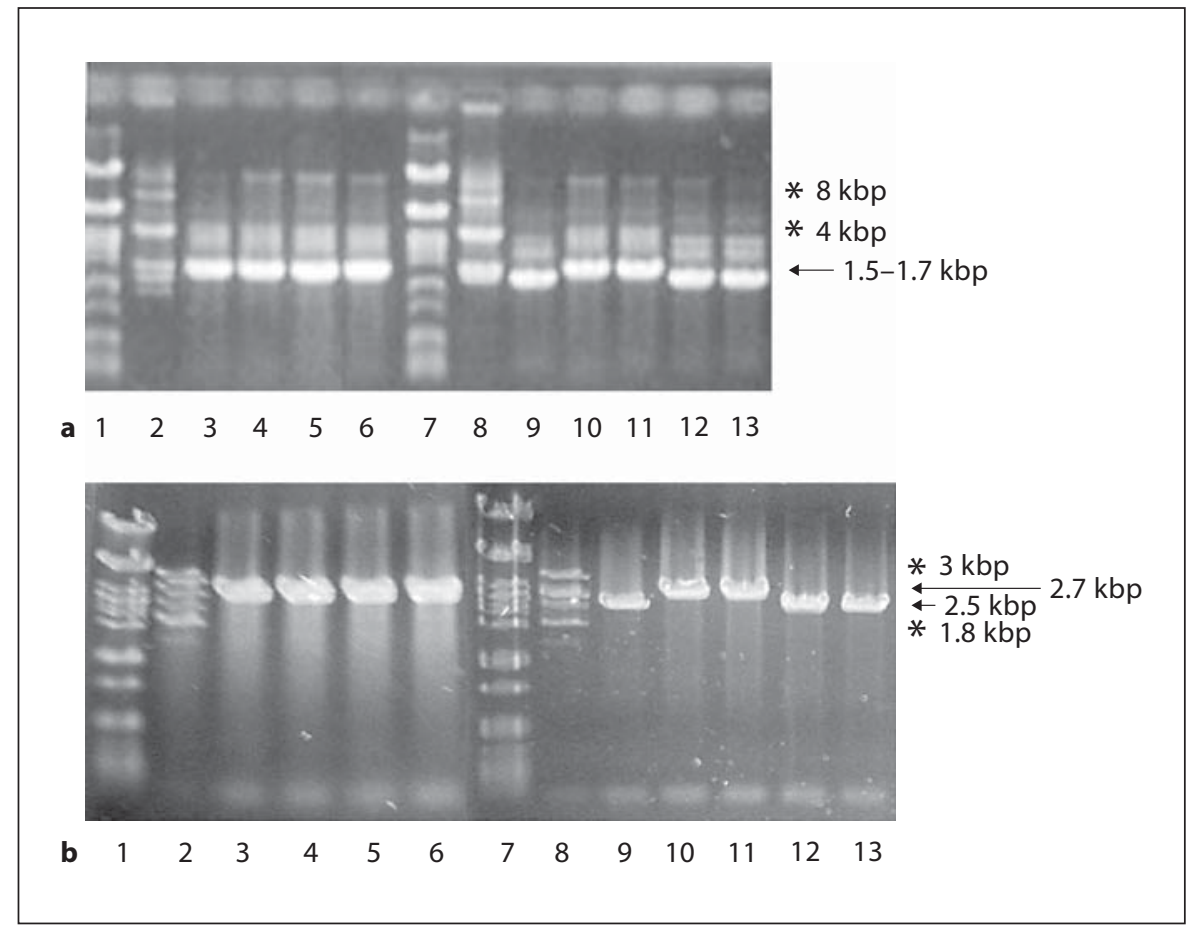

arisen due to specific sequences in pCR-BluntII-TOPO that may act as recognition sequences for the PCV2 Rep protein.

To explain how these plasmids might have arisen, a closer look at PCV replication is necessary. PCV1 and PCV2 replicate their genomes via RCR in a manner similar to Geminiviridae and Nanoviridae but with modifications as described by the RCR melting pot model [7, 17]. After PCV infection of a host cell, cellular DNA polymerases convert the viral ssDNA into a double-stranded DNA (dsDNA) replicative intermediate. Rep and Rep' together bind to the dsDNA at the PCV Ori (fig. 3). The binding site of Rep and Rep' is located at the right leg of a stem-loop and the hexamer repeats (H-repeats) adjacent to it [18]. The $\mathrm{Oc} 8$ sequence $(\mathrm{A} \times \mathrm{TA} \times \mathrm{T} \downarrow \mathrm{AC})$ is located at the apex of the stem-loop (fig. 3a) and is essential for DNA replication $[19,20]$; the arrow indicates the position where the replicase proteins nick the viral DNA strand after binding to the dsDNA replicative intermediate [20, 21]. During viral replication, the replicase proteins remain attached to the nicked viral DNA strand. Cellular DNA polymerases synthesize new DNA and the viral DNA strand is displaced. After DNA synthesis of one genome copy, Rep and Rep' nick the newly synthesized DNA at the Ori to terminate DNA synthesis and Rep and $\mathrm{Rep}^{\prime}$ join the $3^{\prime}-\mathrm{OH}$ and $5^{\prime}-\mathrm{P}$ of the newly synthesized strand [21]. The joining activity of Rep and Rep' at the PCV Ori is aided by base pairing in the stem-loop structure.

Remarkably, Cheung [12] demonstrated that in E. coli, containing 0.8 copy of PCV1 and 0.95 copy of PCV2, with their respective Oris and cloned in tandem, RCR can also take place resulting in the production of PCV circular genomes. It was also shown that this process is governed by the presence of H-repeats and an Oc8 sequence. Production of these circular PCV genomes in E. coli, however, requires the presence of two functional Oris to allow both initiation and termination that result in the formation of a circular genome with one Ori.

However, the original constructs (e.g. infectious clones containing one PCV2 copy) described in the present paper contained one PCV2 Ori and noninterrupted Rep gene. The functionality of the Rep gene was shown by the production of infectious PCV2 virus upon transfection of HindIII-digested infectious clones in PK-15 cells (data not shown). Mutagenesis experiments in the Rep gene lead to the abolishment of the additional DNA species, showing that the truncated plasmids observed in this study result from Rep activity. This suggests that the mutagenized Rep could not initiate RCR at the PCV2 Ori.

Similar to the study by Cheung [12], the single Ori in the original constructs in this study should allow initia- 


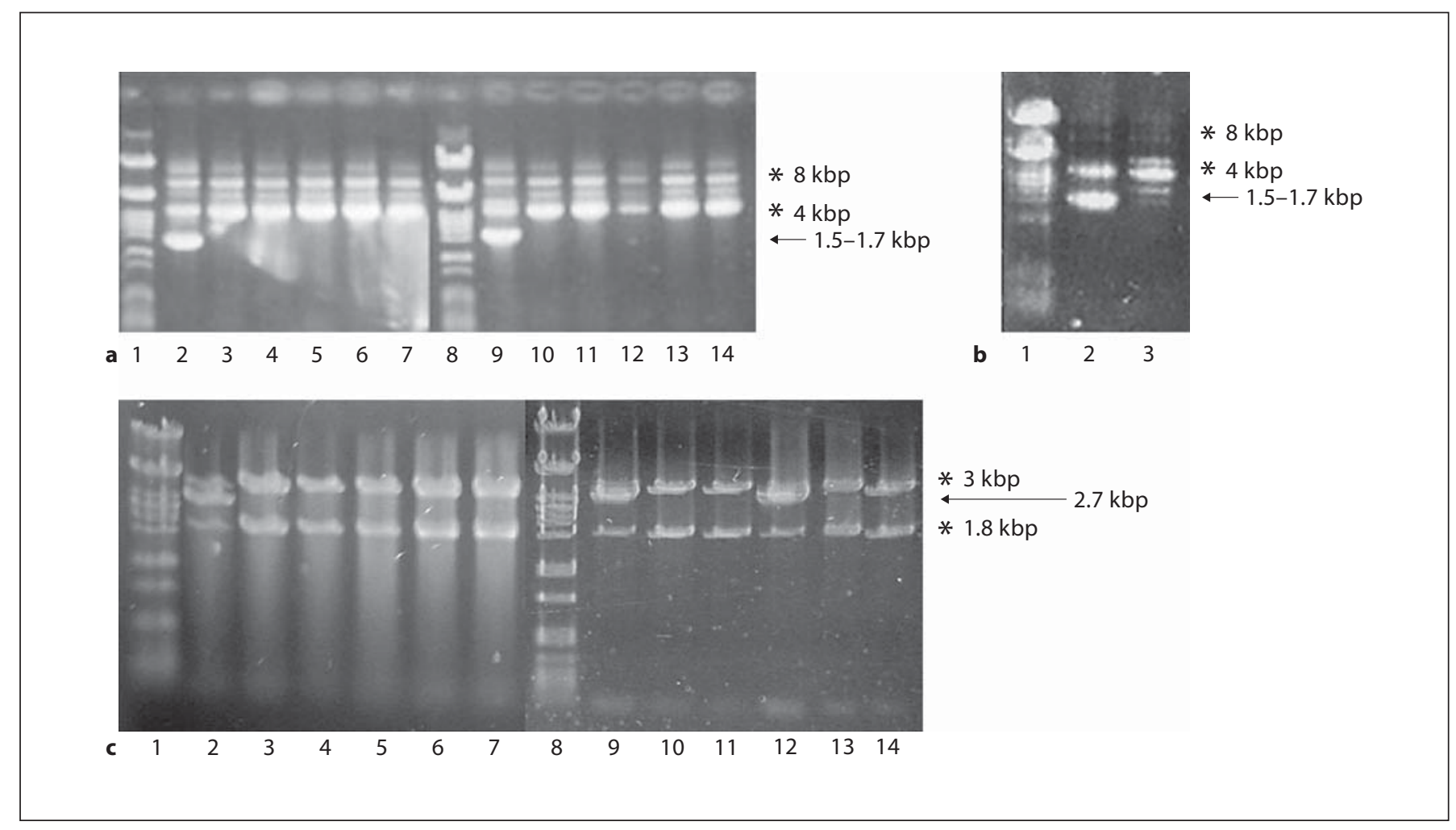

Fig. 6. Agarose gel electrophoresis of plasmid DNA from E. coli containing II9F clone 13 and II9F clone 23 with mutated Rep. a Lanes 1 and 8 : $\lambda$ PstI; lane 2: II9F clone 13; lanes 3-7 contain mutated II9F clone 13 clones. No additional DNA species were present. Lane 9: II9F clone 23; lanes 10-14 contain mutated II9F clone 23 clones, except clone II9F clone 23-3 (lane 12). The latter clone was rerun on a second gel (b). b Lane 2 contains plasmid from II9F clone 23 and lane 3 contains plasmid from II9F clone

tion of replication; however, since only one Ori is present, termination of RCR is expected at the single Ori after one round of DNA synthesis of the PCV2 genome and the plasmid, but the formation of truncated plasmids is not expected. Sequence analysis showed that all truncated plasmids, with the exception of clone TrII9F clone 23, had a nick at the PCV2 Ori, using the standard $\mathrm{A} \times \mathrm{TA} \times \mathrm{T} \downarrow \mathrm{AC}$ Oc8 sequence. TrII9F clone 23 originated from an unusual nick at the base of the right leg of the stem of the PCV2 Ori.

We further asked the question whether Rep could be involved in termination of RCR by e.g. nicking the plasmid in the region upstream of the ZeoR gene and joining the ssDNA ends. The majority of clones showed nicks around position 2050, in the ZeoR promotor and in the 3 ' end of the KanR gene, suggesting that the nicks did not occur due to random strand breaks during the RCR pro-
23-3. Faint additional DNA species of 1.5-1.7 kbp are present in lane 3 (arrow). c HindIII digest of the clones from a. Lanes 1 and 8: $\lambda$ PstI; lane 2: II9F clone 13. Lanes 3-7 contain Rep mutagenized II9F clone 13 clones. No additional 2.7-kbp fragment is observed. Lane 9: II9F clone 23. Lanes 10, 11, 13 and 14 contain Rep mutated II9F clone 23 clones. In lane 12 (Rep nonmutagenized clone II9F 23-3), an additional 2.7-kbp fragment is present.

cess. To our knowledge, it is not known if endonucleases are present in E. coli that could specifically produce single-stranded nicks in this region of the plasmid, or if nicks occur in the ZeoR promotor due to the unwinding of DNA during gene expression. Four clones (Tr4D4 clone 10, Tr4D 4 clone 11, TrII9F clone 13 and TrII9F clone $18 \mathrm{~b})$ had a nick in a region with an inverted and two direct repeats. Previously, it was shown [13] that termination of RCR in E. coli is dependent on the presence of a sequence nonspecific stem-loop structure of which the stem sequence is variable and can be shortened to $7 \mathrm{bp}$. The presence of these kinds of secondary structures in these four clones could be a signal for Rep to nick in this region. In the case of II9F clone $18 \mathrm{a}$, a nick occurred at an Oc8-like sequence in the plasmid (ATTATT $\downarrow G A)$. Cheung [12] showed that the AC nt after the nick in the PCV Oc8 sequence are essential for cleavage by Rep and Rep'. This 
may suggest that the Rep protein may nick at sequences other than the standard Oc8 sequence and is thus promiscuous with regard to its recognition sequences. The reason why no additional bands were observed in clones with PCV2 in the opposite orientation is not known. Although the clones containing the PCV2 in the opposite orientation also contain direct and inverted repeats, apparently the stem-loop structures and/or the sequence context are not appropriate for nicking (by Rep and/or $E$. coli endonuclease).

After nicking in the ZeoR promotor region (by Rep or an $E$. coli endonuclease), joining is necessary to obtain a closed circular ssDNA molecule. Steinfeldt et al. [21] showed in vitro that the joining of ssDNA fragments by Rep and Rep' is favored by base pairing (to bring the $5^{\prime}$ end with attached Rep and Rep' in the proximity of the $3^{\prime}$ end). We did not observe the possibility for strong base pairing between the $5^{\prime}$ end (right part of the PCV2 stem) and the $3^{\prime}$ end of the ssDNA corresponding with the site in the plasmid where nicking had occurred (fig. 3). However, we cannot rule out the possibility that Rep could join the free ends of the ssDNA in the presence of a nick since Steinfeldt et al. [21] showed that joining was not totally abolished in the absence of a stem structure.

The abundance of the truncated plasmids was variable, ranging from low (e.g. 4D4 clone 5) to high (e.g. II9F clone 13). In all cases, E. coli containing the original infectious PCV2 clones were grown under kanamycin selection. Repeated streaking and colony picking (on kanamycin-containing medium) followed by plasmid preparation and gel electrophoresis showed that the abundance of the truncated plasmids was variable, e.g. some clones contained both the original and truncated plasmid while other clones mainly contained the truncated plasmid (data not shown). Since it was shown that the KanR gene was not present in the truncated plasmids, this raises the question if the truncated plasmids, which contain an Ori for $\theta$-replication, were further replicated in E. coli either via $\theta$-replication or RCR, or continuously newly produced from the original clone. Previously, Cheung [12] suggested that - in E. coli - plasmids containing a PCV Ori (but not flanked by an inverted repeat) and an Ori for $\theta$-replication, were replicated via $\theta$-replication. In this study, it was observed that the truncated plasmids TrII9F clone 10, TrII9F clone 18a and TrII9F clone 18b, containing an origin for $\theta$-replication, were stably maintained after transformation in E. coli. In addition, these truncated plasmids did not contain the region upstream of the nick in the PCV2 Ori, which is part of the Rep gene promoter and, therefore, makes it unlikely that transcription of the Rep gene occurred. Together, this suggests that the truncated plasmids could replicate in $E$. coli via $\theta$-replication.

In conclusion, the presence of uncommon, additional DNA species in E. coli harboring a plasmid with one PCV2 genome copy was shown. These DNA species were truncated forms of the original plasmid containing the PCV2 genome. Data presented here show that these species have arisen due to the activity of the Rep gene in E. coli and the presence of a PCV2 Ori and probably plasmid specific sequences that can be used by the Rep gene for nicking and termination of RCR. Although Rep was involved in initiation of replication of these truncated DNA plasmids, it could not be demonstrated that Rep was also involved in termination of replication. If Rep was also involved in termination, this would suggest that Rep nicking can occur at non-Oc8 sites in the presence of (a) secondary structure(s), shedding a new light on Rep activity.

\section{Acknowledgements}

The authors acknowledge Carine Boone, Hanne Van Gorp and Dries Helderweirt for their excellent technical assistance. P.L.D. is a postdoctoral fellow of FWO-Vlaanderen.

\section{References}

1 Pringle CR: Virus taxonomy at the XIth International Congress of Virology, Sydney, Australia, 1999. Arch Virol 1999;144:20652070.

2 Hamel AL, Lin LL, Nayar GP: Nucleotide sequence of porcine circovirus associated with postweaning multisystemic wasting syndrome in pigs. J Virol 1998;72:5262-5267.

3 Meehan BM, McNeilly F, Todd D, Kennedy S, Jewhurst VA, Ellis JA, Hassard LE, Clark EG, Haines DM, Allan GM: Characterization of novel circovirus DNAs associated with wasting syndromes in pigs. J Gen Virol 1998;79:2171-2179.

4 Morozov I, Sirinarumitr T, Sorden SD, Halbur PG, Morgan MK, Yoon KJ, Paul PS: Detection of a novel strain of porcine circovirus in pigs with postweaning multisystemic wasting syndrome. J Clin Microbiol 1998;36: 2535-2541.

5 Cheung AK: The essential and nonessential transcription units for viral protein synthesis and DNA replication of porcine circovirus type 2. Virology 2003;313:452-459. 
6 Nawagitgul P, Morozov I, Bolin SR, Harms PA, Sorden SD, Paul PS: Open reading frame 2 of porcine circovirus type 2 encodes a major capsid protein. J Gen Virol 2000;81:22812287.

$\checkmark 7$ Cheung AK: Detection of template strand switching during initiation and termination of DNA replication of porcine circovirus. J Virol 2004;78:4268-4277.

8 Lekcharoensuk P, Morozov I, Paul PS, Thangthumniyom N, Wajjawalku W, Meng $\mathrm{XJ}$ : Epitope mapping of the major capsid protein of type 2 porcine circovirus (PCV2) by using chimeric PCV1 and PCV2. J Virol 2004;78:8135-8145.

$\checkmark 9$ Liu C, Wei Y, Znahg C, Lu Y, Kong X: Construction and characterization of porcine circovirus type 2 carrying a genetic marker strain. Virus Res 2007;127:95-99.

10 Grasland B, Loizel C, Blanchard P, Oger A, Nignol A-C, Bigarré L, Morvan H, Cariolet $\mathrm{R}$, Jestin A: Reproduction of PMWS in immunostimulated SPF piglets transfected with infectious cloned genomic DNA of type 2 porcine circovirus. Vet Res 2005;36:685697.
1 Roca M, Balasch M, Segalés J, Calsamiglia M, Viaplana E, Urniza A, Hattermann K, Mankertz A, Plana-Durán J, Domingo M: In vitro and in vivo characterization of an infectious clone of a European strain of porcine circovirus type 2. J Gen Virol 2004;85: 1259-1266.

12 Cheung AK: Rolling-circle replication of an animal circo circovirus genome in a thetareplicating bacterial plasmid in Escherichia coli. J Virol 2006;80:8686-8694.

13 Cheung AK: A stem-loop structure, sequence non-specific, at the origin of DNA replication of porcine circovirus is essential for termination but not for initiation of rolling-circle DNA replication. Virology 2007; 363:229-235.

14 Lefebvre DJ, Van Doorsselaere J, Delputte PL, Nauwynck HJ: Recombination of two porcine circovirus type 2 strains. Arch Virol 2009; 154:875-879.

15 Lefebvre DJ, Costers S, Van Doorsselaere J, Misinzo G, Delputte PL, Nauwynck HJ: Antigenic differences among porcine circovirus type 2 strains, as demonstrated by the use of monoclonal antibodies. J Gen Virol 2008;89: 177-187.
16 Steinfeldt T, Finsterbusch T, Mankertz A: Functional analysis of cis- and trans-acting replication factors of porcine circovirus type 1. J Virol 2007;81:5696-5704.

17 Cheung AK: Palindrome regeneration by template strand-switching mechanism at the origin of DNA replication of porcine circovirus via the rolling-circle melting-pot replication model. J Virol 2004;78:9016-9029.

18 Steinfeldt T, Finsterbusch T, Mankertz A: Rep and Rep' protein of porcine circovirus type 1 bind to the origin of replication in vitro. Virology 2001;291:152-160.

19 Cheung AK: Identification of an octanucleotide motif sequence essential for viral protein, DNA, and progeny virus biosynthesis at the origin of DNA replication of porcine circovirus type 2. Virology 2004;324:28-36.

20 Cheung AK: Detection of rampant nucleotide reversion at the origin of DNA replication of porcine circovirus type 1. Virology 2005;333:22-30.

21 Steinfeldt T, Finsterbusch T, Mankertz A: Demonstration of nicking/joining activity at the origin of DNA replication associated with the Rep and Rep' proteins of porcine circovirus type 1. J Virol 2006;80:62256234. 